\title{
The Homology of the Real Complement of a $k$-parabolic Subspace Arrangement
}

\author{
Christopher Severs ${ }^{1}$ and Jacob A. White ${ }^{2}$ \\ ${ }^{1}$ Mathematical Sciences Research Institute, Berkeley, $C A$ \\ ${ }^{2}$ School of Mathematical and Statistical Sciences, Arizona State University, Tempe, AZ
}

\begin{abstract}
The $k$-parabolic subspace arrangement, introduced by Barcelo, Severs and White, is a generalization of the well known $k$-equal arrangements of type- $A$ and type- $B$. In this paper we use the discrete Morse theory of Forman to study the homology of the complements of $k$-parabolic subspace arrangements. In doing so, we recover some known results of Björner et al. and provide a combinatorial interpretation of the Betti numbers for any $k$-parabolic subspace arrangement. The paper provides results for any $k$-parabolic subspace arrangement, however we also include an extended example of our methods applied to the $k$-equal arrangements of type- $A$ and type- $B$. In these cases, we obtain new formulas for the Betti numbers.
\end{abstract}

Résumé. L'arrangement k-parabolique, introduit par Barcelo, Severs et White, est une généralisation des arrangements, k-éguax de type A et de type B. Dans cet article, nous utilisons la théorie de Morse discrète proposée par Forman pour étudier l'homologie des compléments d'arrangements k-paraboliques. Ce faisant, nous retrouvons les résultats connus de Bjorner et al. mais aussi nous fournissons une interprétation combinatoire des nombres de Betti pour des arrangements k-paraboliques. Ce papier fournit alors des résultats pour n'importe quel arrangement kparabolique, cependant nous y présentons un exemple étendu de nos méthodes appliquées aux arrangements k-éguax de type A et de type B. Pour ce cas, on obtient de nouvelles formules pour les nombres de Betti.

Keywords: Subspace Arrangements, Discrete Morse Theory, Coxeter Groups

\section{Introduction}

Recall that the real (essentialized) $k$-equal subspace arrangement, $\mathcal{A}_{n, k}$ is defined to be the collection of subspaces given by

$$
x_{i_{1}}=x_{i_{2}}=\cdots x_{i_{k}} \text { and subject to the relation } \sum_{i=1}^{n+1} x_{i}=0 .
$$

This arrangement was originally studied by Björner and Lovász in connection with linear decision trees and the $k$-equal problem [3]. In particular, they showed how the Betti numbers could be used to give lower bounds on the number of leaves in a decision tree which solves the $k$-equal problem. The cohomology of the complement of this subspace arrangement was studied by Björner and Welker in [6]. The Betti numbers have applications in the study of linear decision trees and the $k$-equal problem. Recall that the 
complement of a subspace arrangement $\mathcal{A}$ is $M_{\mathcal{A}}=\mathbb{R}^{n}-\cup_{X \in \mathcal{A}} X$. In order to compute the cohomology group of the complement they make use of the well known Goresky-MacPherson formula for subspace arrangements [8].

Proposition 1.1 (Goresky-MacPherson Formula) Let $\mathcal{A}$ be subspace arrangement with complement $M_{\mathcal{A}}$, and let $\mathcal{L}_{\mathcal{A}}$ be the intersection lattice of $\mathcal{A}$. Then

$$
\widetilde{H}^{i}\left(M_{\mathcal{A}}\right) \cong \bigoplus_{x \in \mathcal{L} \geq 0} \widetilde{H}_{\operatorname{codim}(x)-i-2}(\Delta(\hat{0}, x))
$$

where $\Delta(\hat{0}, x)$ is the order complex of the interval between $\hat{0}$ and $x$.

By considering the homology of the intersection lattice of $\mathcal{A}_{n, k}$, Björner and Welker were able to give a description of the cohomology of the complement, $M_{\mathcal{A}_{n, k}}$. Later, Björner and Wachs [5] used lexicographic shellability to study the intersection lattice $\mathcal{L}_{\mathcal{A}_{n, k}}$.

This method of studying subspace arrangements via the Goresky-MacPherson formula and shellability of the intersection lattice was continued in the work of Björner and Sagan [4]. In this work they define type- $B$ and type- $D$ analogues of the $k$-equal subspace arrangement. They then show that the intersection lattice in the case of type- $B$ is shellable and prove results about the cohomology of the complement of the type- $B k$-equal arrangement using the Goresky-MacPherson formula.

The cohomology of complement of the type- $D k$-equal arrangement was further studied by Feichtner and Kozlov. In [7], they prove that the intersection lattice of the type- $D k$-equal subspace arrangement is shellable for large values of $k$ and again use the Goresky-MacPherson formula to calculate the cohomology groups in those cases. Their approach uses a generalization of lexicographic shellability due to Kozlov [10]. For smaller values of $k$, Feichtner and Kozlov use more sophisticated techniques from algebraic topology to approach the problem.

In this paper, we revisit the study of the complement of the $k$-equal arrangements of type- $A, B$ and $D$. via a generalization known as $k$-parabolic subspace arrangements. Introduced by Barcelo, Severs and White in [2], the $k$-parabolic arrangement is a real subspace arrangement that may be defined for any finite real reflection group, $W$. The subspaces in the arrangement are those which are fixed under the action of irreducible parabolic subgroups of $W$ that have rank $k-1$. More information on these arrangements is provided in Section 2

Our study of the homology of the complement of the $k$-parabolic subspace arrangement is not done via the Goresky-MacPherson formula and the intersection lattice, but rather by using the discrete Morse theory of Forman. First we construct a polyhedral complex $\Delta_{k}(W)$ that is homotopy equivalent to the complement of the $k$-parabolic arrangement. Recall that the poset of all cosets of parabolic subgroups under inclusion is isomorphic to the face lattice of the Coxeter arrangement corresponding to $W$. We obtain the face poset for $\Delta_{k}(W)$ by removing the upper order ideals corresponding to the irreducible parabolic subgroups of rank $k-1$. We then create an acyclic matching on this poset and use discrete Morse theory to calculate the homology groups and Betti numbers for $\Delta_{k}(W)$, and hence the complement.

The remainder of the paper is divided into three sections. In the first section we recall some definitions and theorems that we will need concerning $k$-parabolic subspace arrangements and discrete Morse theory. In the next section we provide an extended example of our methods using the type- $A$ and type- $B k$-equal subspace arrangements. These arrangements should be familiar to most readers and give a more intuitive look at our matching and study of the homology of the complement. Moreover, we obtain new formulas 
for the Betti numbers of these arrangements, along with a new combinatorial interpretation for them. In the final section we present our matching in fullest generality as well, including some periodicity results for the homology, and a combinatorial interpretation of the Betti numbers. We also mention some open questions.

\section{Background}

The material on $k$-parabolic arrangements in this section is taken from [2]. The definitions and theorems concerning discrete Morse theory are primarily from Kozlov's Combinatorial Algebraic Topology [9], but may also be found in many other papers and books. This section contains no new material.

\section{$2.1 \quad k$-parabolic subspace arrangements}

We start with the definition of the $k$-parabolic arrangement $\mathcal{W}_{n, k}$. Choose a finite real reflection group $W$ of rank $n$ with simple reflections $S$. A parabolic subgroup is a subgroup of the form $w\langle I\rangle w^{-1}$, for some $I \subseteq S, w \in W$. Consider $\mathcal{P}(W)$, the lattice of all parabolic subgroups of $W$. It was shown by Barcelo and Ihrig [1] that the intersection lattice of the Coxeter arrangement associated to $W, \mathcal{L}(\mathcal{H}(W))$, is isomorphic to $\mathcal{P}(W)$. The isomorphism is established by sending a parabolic subgroup to the set of points in $\mathbb{R}^{n}$ that it fixes, and sending an intersection of hyperplanes in the Coxeter arrangement to the parabolic subgroup of $W$ that fixes the points in the intersection. We will use this correspondence to define a $k$-parabolic subspace arrangement associated to $W$.

Definition 2.1 ( $k$-parabolic subspace arrangement) Let $W$ be a finite real reflection group of rank $n$ and let $\mathcal{P}_{n, k}(W)$ be the lattice of all irreducible parabolic subgroups of $W$ of rank $k-1$. The $k$-parabolic subspace arrangement, $\mathcal{W}_{n, k}$ is the collection of subspaces

$$
\left\{F i x(G) \mid G \in \mathcal{P}_{n, k}(W)\right\}
$$

As with the $k$-equal arrangement, the $k$-parabolic arrangement can be embedded in its corresponding Coxeter arrangement. This allows us to think of the subspaces in $\mathcal{W}_{n, k}$ as intersections of hyperplanes of the Coxeter arrangement. Also, $\mathcal{L}\left(\mathcal{W}_{n, k}\right)$ is a subposet of $\mathcal{L}(\mathcal{H}(W))=\mathcal{L}\left(\mathcal{W}_{n, 2}\right)$.

If we let $W=A_{n}$ then it is easy to see that we recover the $k$-equal subspace arrangement from the introduction. Furthermore, if we let $W=B_{n}$, we recover the $\mathcal{B}_{n, k, k-1}$ subspace arrangements of Björner and Sagan. We note however when $W=D_{n}$, we do not recover the $\mathcal{D}_{n, k}$ arrangements of Björner and Sagan except in the special case $k=3$.

The natural embedding of $\mathcal{W}_{n, k}$ into the Coxeter arrangement allows us to use a construction, due to Orlik [11], to create a cell complex homotopy equivalent to the complement. To be more precise, there is a order-reversing correspondence between the face poset of the Coxeter arrangement and the dual zonotope, known as the $W$-Permutahedron. Under this correspondence, removing a subcomplex of faces of $\mathcal{W}_{n, k}$ is homotopy equivalent to a polyhedral subcomplex of the Permutahedron. In the case of the complement $M_{\mathcal{W}_{n, k}}$, we apply this fact to the subcomplex generated by all faces contained in a subspace of $\mathcal{W}_{n, k}$. We shall call the resulting cell complex $\Delta_{k}(W)$.

Lemma 2.2 There exists a polyhedral complex, $\Delta_{k}(W)$, such that $\Delta_{k}(W)$ is homotopy equivalent to $M_{\mathcal{W}_{n, k}}$. Moreover, the face poset of $\Delta_{k}(W)$ is a subposet of the poset of cosets of parabolic subgroups of $W$, ordered by inclusion. It is obtained by removing all upper order ideals generated by any $u G$, where $G$ is an irreducible parabolic subgroup of rank $k-1$. 


\subsection{Discrete Morse theory}

Robin Forman's discrete Morse theory provides a means to calculate the homology groups of a simplicial or CW complex by studying the face poset of the complex. Informally, the goal is to find an acyclic matching on the face poset of the complex. Then the main results of discrete Morse theory state that the original complex is homotopy equivalent to a complex that has one cell of dimension $i$ for each unmatched element in the poset on level $i-1$. We will see that this is especially useful if there are unmatched elements on only one level, or if there is a gap between the levels which share unmatched elements. In these cases, we are able to easily calculate the homology groups of the complex.

We begin by presenting the definition of an acyclic matching that we will use.

Definition 2.3 ([9], Definition 11.1) Let $P$ be a poset.

1. A partial matching in $P$ is a partial matching in the underlying graph of the Hasse diagram of $P$, i.e., it is a subset $M \subseteq P \times P$ such that

- $(a, b) \in M$ implies $b \succ a$ ( $b$ covers $a)$;

- each $a \in P$ belongs to at most one element in $M$.

When $(a, b) \in M$ we write $a=d(b)$ and $b=u(a)$.

2. A partial matching on $P$ is called acyclic if there does not exist a cycle

$$
b_{1} \succ d\left(b_{1}\right) \prec b_{2} \succ d\left(b_{2}\right) \prec \cdots \prec b_{n} \succ d\left(b_{n}\right) \prec b_{1}
$$

with $n>2$ and all $b_{i} \in P$ being distinct.

The second condition may be thought of in the following way. Consider the Hasse diagram of $P$ as a directed graph, with all of the edges oriented downwards, from larger to smaller. Now, if $(a, b) \in M$, we change the orientation of the edge connecting $a$ and $b$. The matching is called acyclic if the directed graph obtained in the above manner is acyclic. Oftentimes the Cluster Lemma, or Patchwork Theorem, is used to construct acyclic matchings.

Lemma 2.4 ([9], Theorem 11.10) Assume that $\varphi: P \rightarrow Q$ is an order-preserving map, and assume that we have acyclic matchings on subposets $\varphi^{-1}(q)$ for all $q \in Q$. Then the union of these matchings is itself an acyclic matching on $P$.

The proof that our matching for the $k$-parabolic arrangements is acyclic will rely on Lemma 2.4 . In application, one tries to break a poset $P$ into 'smaller' pieces, and place an acyclic matching on these pieces. In our case, we will give the matching, and then show that it restricts to fibers, to help simplify the proof that the matching is acyclic.

Given an acyclic matching $M$ on a poset $P$, we say the elements in $P \backslash M$ are critical. The fundamental theorem of discrete Morse theory states that the critical elements correspond to the cells or simplices of a new complex that the original complex is homotopy equivalent to.

Theorem 2.5 ([9], Theorem 11.13) Let $\Delta$ be a polyhedral complex, and let $M$ be an acyclic matching on $\mathcal{F}(\Delta) \backslash\{\hat{0}\}$. Let $c_{i}$ denote the number of critical $i$-dimensional cells of $\Delta$. 
(a) If the critical cells form a subcomplex $\Delta_{c}$ of $\Delta$, then there exists a sequence of cellular collapses leading from $\Delta$ to $\Delta_{c}$.

(b) In general, the space $\Delta$ is homotopy equivalent to $\Delta_{c}$, where $\Delta_{c}$ is a CW complex with $c_{i}$ cells in dimension $i$.

(c) There is a natural indexing of cells of $\Delta_{c}$ with the critical cells of $\Delta$ such that for any two cells $\sigma$ and $\tau$ of $\Delta_{c}$ satisfying $\operatorname{dim} \sigma=\operatorname{dim} \tau+1$, the incidence number $[\tau: \sigma]$ is given by

$$
[\tau: \sigma]=\sum_{c} w(c)
$$

Here the sum is taken over all alternating paths $c$ connecting $\sigma$ with $\tau$, i.e., over all sequences $c=$ $\left(\sigma, a_{1}, u\left(a_{1}\right), \ldots, a_{t}, u\left(a_{t}\right), \tau\right)$ such that $\sigma \succ a_{1}, u\left(a_{t}\right) \succ \tau$, and $u\left(a_{i}\right) \succ a_{i+1}$, for $i=1, \ldots, a_{t-1}$. For such an alternating path, the quantity $w(c)$ is defined by

$$
w(c):=(-1)^{t}\left[a_{1}: \sigma\right]\left[\tau: u\left(a_{t}\right)\right] \prod_{i=1}^{t}\left[a_{i}: u\left(a_{i}\right)\right] \prod_{i=1}^{t-1}\left[a_{i+1}: u\left(a_{i}\right)\right]
$$

where the incidence numbers in the right-hand side are taken in the complex $\Delta$.

\section{The type- $A$ and type- $B k$-equal arrangements}

In this section we give two examples of our usage of discrete Morse theory on $k$-parabolic subspace arrangements. The examples we have chosen, the type- $A k$-equal arrangement $\left(\mathcal{A}_{n, k}\right)$ and the type- $B k$ equal arrangement $\left(\mathcal{B}_{n, k, k-1}\right)$ have been studied and should be familiar to many readers. We have chosen to present these two examples first because we believe they give the most intuitive look at our matching, and because our matching can be used to obtain new results regarding these arrangements.

\subsection{Acyclic matching and homology results for the $k$-equal arrangement}

We start with the type- $A k$-equal arrangement. This arrangement, described in the introduction, is embedded in the Coxeter arrangement, $\mathcal{H}\left(A_{n}\right)$. It is well known that the face lattice $\mathcal{F}\left(\mathcal{H}\left(A_{n}\right)\right)$ may be thought of as the poset of all set compositions of $[n+1]$ with reverse refinement as the partial order.

We need to obtain a combinatorial description of the face lattice of $\Delta_{k}\left(A_{n}\right)$. Since the face poset of the Permutahedron is dual to $\mathcal{F}\left(\mathcal{H}\left(A_{n}\right)\right)$, first we reverse the partial order. Then we consider faces of the Permutahedron whose corresponding set compositions have a block of size $k$ and remove the upper order ideal of these elements. This will leave us with a subposet of $\mathcal{F}\left(\mathcal{H}\left(A_{n}\right)\right)$ in which all elements have blocks of size at most $k-1$. In order to construct a matching on the face poset of the complement, $\mathcal{F}\left(\Delta_{k}(W)\right)$ we need the following definition.

Definition 3.1 Given two sets $S, T \in[n+1]$ with $S \cap T=\emptyset$, we say that there is a descent from set $S$ to set $T$ if $\max (S)>\min (T)$. Otherwise we say there is an ascent from $S$ to $T$.

We are now ready to construct a matching $M$ on $\mathcal{F}\left(\Delta_{k}(W)\right)$. The matching is given by the following algorithm: Given an element $\left(B_{1}, B_{2}, \ldots, B_{t}\right)$ we consider pairs of adjacent blocks $B_{i}$ and $B_{i+1}$. We start with $i=1$. 
1. If $B_{i}$ is not a singleton, we match

$$
\left(B_{1}, \ldots, B_{i}, B_{i+1}, \ldots, B_{t}\right)
$$

with

$$
\left(B_{1}, \ldots, B_{i-1},\left\{\min \left(B_{i}\right)\right\}, B_{i} \backslash\left\{\min \left(B_{i}\right)\right\}, \ldots, B_{t}\right) .
$$

2. If there is a descent from $B_{i}$ to $B_{i+1}$, we set $i=i+1$ and start over at step one.

3. If $\left|B_{i+1}\right|=k-1$, then we set $i=i+2$ and start over at step one.

4. We match

$$
\left(B_{1}, \ldots, B_{i}, B_{i+1}, \ldots, B_{t}\right)
$$

with the element

$$
\left(B_{1}, \ldots, B_{i} \cup B_{i+1}, \ldots, B_{t}\right) .
$$

Note that it is only possible to match elements that differ by the addition or removal of a singleton and hence are on adjacent levels of the poset. The algorithm finishes when a match is found or we reach $i=t$. In the latter case, we have identified a critical element.

Proposition 3.2 The matching $M$ described above is acyclic.

The result follows from the general case, Proposition 4.1. The elements that are unmatched have a series of singletons with a descent between each adjacent pair, followed by an ascent to a size $k-1$ block, followed by a series of singletons with a descent between each adjacent pair, followed by an ascent to a size $k-1$ block, etc. An example of the matching along with some critical elements is shown in Figure 1 . In the example, there are three elements on the same level of the poset. The first is matched with an element above by merging two blocks. The second is matched with an element below by splitting a block, and the third is a critical element. In the example, $n=8$ and $k=4$.

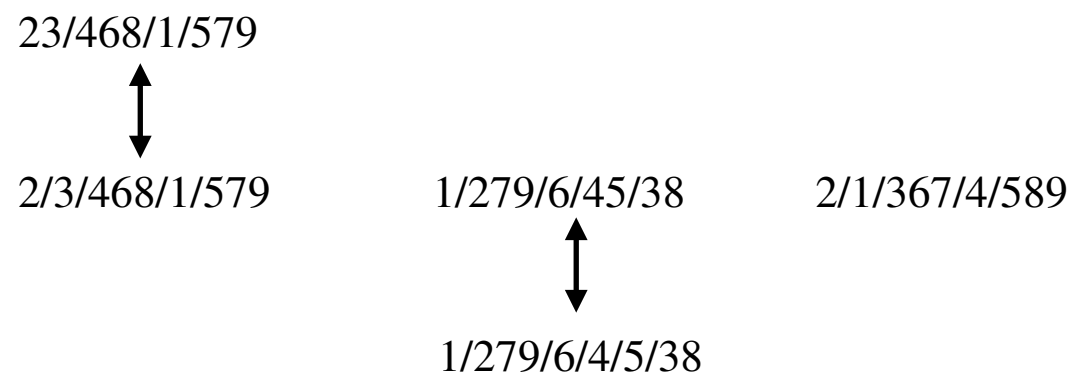

Fig. 1: A matching between elements in $\Delta_{4}\left(A_{8}\right)$

Also note that critical elements may only occur on levels that are a multiple of $k-2$. By Theorem 2.5 . we can already conclude that $H_{i}\left(M_{\mathcal{A}_{n, k}}\right)$ is trivial when $i$ is not a multiple of $k-2$. In the case where $k>3$ we also know that the non-trivial homology groups are free. We see this in the following way. Suppose there are $c_{j(k-2)}$ critical elements on level $j(k-2)$. These correspond to cells of dimension $j(k-2)$ 
in a CW complex that is homotopy equivalent to $M_{\mathcal{A}_{n, k}}$. Furthermore, there are no critical elements on levels $j(k-2)-1$ or $j(k-2)+1$ and hence no cells of these dimensions. Thus, we have that the chain groups $C_{j(k-2)-1}$ and $C_{j(k-2)+1}$ are trivial and $C_{j(k-2)}$ is free abelian of rank $c_{j(k-2)}$. The boundary maps $\partial_{j(k-2)+1}$ and $\partial_{j(k-2)}$ can only be the trivial map, which implies $H_{j(k-2)}\left(M_{\mathcal{A}_{n, k}}\right) \cong C_{j(k-2)}$.

It remains to calculate the number of critical cells at each level. Let $j$ be an integer such that $0 \leq j \leq$ $n / k$. Then the number of unmatched cells in dimension $j(k-2)$ is given by:

$$
\sum_{\substack{i_{0}+\cdots+i_{j}=n \\
i_{m} \geq k, \forall 1 \leq m \leq j}}\left(\begin{array}{c}
n \\
i_{0}, \ldots, i_{j}
\end{array}\right) \prod_{m=1}^{j}\left(\begin{array}{c}
i_{m}-1 \\
k-1
\end{array}\right)
$$

where the sum is over all integer compositions of $n$ into $j+1$ parts, such that each part, with the exception of the first part, has size at least $k$. In all other dimensions there are no critical cells. The formula comes from the following: consider a composition of $[n]$ into $j+1$ parts whose sizes are given by $i_{0}, \ldots, i_{k}$. For each block, besides the first one, take $k-1$ elements that are not the minimum of that part. Make this a block, and place all other elements of that block as singletons in descreasing order. Finally partition the first block into singletons and append them to the end of the composition in decreasing order. Clearly this gives all set compositions that meet our criteria for not being matched.

Combining the results above, Theorem 2.5 and Lemma 2.2, we have the following.

Theorem 3.3 The homology groups $H_{i}\left(M_{\mathcal{A}_{n, k}}\right)$ are non-trivial only when $i=j(k-2)$, for $j \leq\left\lfloor\frac{n}{k}\right\rfloor$. Furthermore, $H_{j(k-2)}\left(M_{\mathcal{A}_{n, k}}\right)$ is free abelian of rank

$$
\sum_{\substack{i_{0}+\cdots+i_{j}=n \\
i_{m} \geq k, \forall 1 \leq m \leq j}}\left(\begin{array}{c}
n \\
i_{0}, \ldots, i_{j}
\end{array}\right) \prod_{m=1}^{j}\left(\begin{array}{c}
i_{m}-1 \\
k-1
\end{array}\right),
$$

where the sum is over all integer compositions of $n$ into $j+1$ parts, such that each part, with the exception of the first part, has size at least $k$.

Note that the above formula is new, and simpler than previous formulas obtained. The case $j=1$ specializes to a formula previously known by Björner and Welker [6]. The case where $k=3$ is the most difficult case. We will discuss this case for general $W$ later.

\subsection{Acyclic matching and homology results for $\mathcal{B}_{n, k}$}

We now turn to the type- $B k$-equal arrangement, $\mathcal{B}_{n, k, h}$. The arrangement $\mathcal{B}_{n, k, h}$ has subspaces given by

$$
\pm x_{i_{1}}=\cdots= \pm x_{i_{k}} \text { as well as } x_{j_{1}}=\cdots=x_{j_{h}}=0
$$

This arrangement is embedded in the type- $B$ Coxeter arrangement, $\mathcal{H}\left(B_{n}\right)$, and the face lattice $\mathcal{F}\left(\mathcal{H}\left(B_{n}\right)\right)$ has a description in terms of set compositions of $\{0,1, \ldots, n, \overline{1}, \ldots, \bar{n}\}$. For technical reasons, we will use the linear order $\bar{n}<\cdots<\overline{1}<0<1<\cdots<n$.

A type- $B$ set composition consists of a set composition of $[0, n]$ such that 0 is in the first block (henceforth called the zero block). The numbers in the 0 block are all unbarred, and in the non-zero blocks we may replace $i$ with $\bar{i}$. The order is reverse refinement, and when blocks are merged the bars do not change, 
except if a block is merged with the zero block. In this latter case all elements become unbarred. We again say there is a descent from $B_{i}$ to $B_{i+1}$ if $\max \left(B_{i}\right)>\min \left(B_{i+1}\right)$.

As in the type- $A$ case, we obtain the face poset, $\mathcal{F}\left(\Delta_{k}\left(B_{n}\right)\right)$, by removing all type- $B$ set compositions that have blocks, including the zero block, of size $k$ or greater. Thus we only study type- $B$ set compositions with blocks of size at most $k-1$. Recall that we also reverse the partial order, so now the partial order is refinement.

The matching algorithm that we give is sort of dual to the one in the type- $A$ case. Instead we start at the last block and work our way towards the zero block. Given an element $\left(B_{0}, B_{1}, B_{2}, \ldots, B_{t}\right)$ we consider pairs of adjacent blocks $B_{i}$ and $B_{i+1}$. We start with $i=t$.

1. If $B_{i}$ is not a singleton, we match

$$
\left(B_{0}, \ldots, B_{i}, B_{i+1}, \ldots, B_{t}\right)
$$

with

$$
\left(B_{0}, \ldots, B_{i-1},\left\{B_{i} \backslash\left\{\max \left(B_{i}\right)\right\},\left\{\max \left(B_{i}\right)\right\}, \ldots, B_{t}\right) .\right.
$$

2. If there is a descent from $B_{i-1}$ to $B_{i}$, we set $i=i-1$ and start over again at step one.

3. If $\left|B_{i-1}\right|=k-1$, then we set $i=i-2$ and start over again at step one.

4. We match

$$
\left(B_{0}, \ldots, B_{i-1}, B_{i}, \ldots, B_{t}\right)
$$

with the element

$$
\left(B_{0}, \ldots, B_{i-1} \cup B_{i}, \ldots, B_{t}\right) .
$$

Again the above algorithm gives an acyclic matching on $\mathcal{F}\left(\Delta_{k}\left(B_{n}\right)\right)$. Critical cells have the following properties:

- all blocks are singletons or have size $k-1$,

- every block of size $k-1$ is followed by a singleton, with an ascent between them,

- every pair of adjacent singletons forms a descent.

Again, as in the type- $A$ case, when $k>3$ we have non-trivial free abelian homology groups only in period $k-2$. We also note here that our restriction of $h=k-1$ may be removed and the matching will work with any $h$. Removing this restriction takes us out of the class of $k$-parabolic subspace arrangements and covers all of the $\mathcal{B}_{n, k, h}$ arrangements defined by Björner and Sagan.

The only modification to the matching algorithm is that when considering whether we may merge a singleton and the zero block we check to see if the zero block is of size $<h$, rather than $<k-1$. The condition for being a critical cell involves checking if the 0-block has size 1 or $h$, rather than $k-1$.

For $h=k$ or $h=2$, we run into the same issues as the $k=3$ case, namely that we end up with critical elements on adjacent levels of the poset. In the case where $h \neq 2, k$ it is easy to see that the non-trivial homology groups are free abelian and appear in period $t(k-2)$ and $t(k-2)+h-1$, recovering the periodicity results obtained by Björner and Sagan [4]. Moreover, by using a counting argument similar to the type- $A$ case, we obtain a new formula for the Betti numbers. Using the same reasoning as in the type- $A$ case, we obtain the following result. 
Theorem 3.4 The homology groups $H_{i}\left(M_{\mathcal{B}_{n, k}}\right)$ are non-trivial only when $i=j(k-2)$, for $j \leq\left\lfloor\frac{n}{k}\right\rfloor$. Furthermore, $H_{j(k-2)}\left(M_{\mathcal{B}_{n, k}}\right)$ is free abelian of rank

$$
\sum_{\substack{i_{0}+\ldots+i_{j}=n \\
i_{m} \geq k, \forall 1 \leq m \leq j}}\left(\begin{array}{c}
n \\
i_{0}, \ldots, i_{j}
\end{array}\right) \prod_{m=1}^{j} 2^{i_{m}}\left(\begin{array}{c}
i_{m}-1 \\
k-1
\end{array}\right)+\sum_{\substack{i_{0}+\ldots+i_{j}=n \\
i_{1} \geq k-1 \\
i_{m} \geq k, \forall 2 \leq m \leq j}}\left(\begin{array}{c}
n \\
i_{0}, \ldots, i_{j}
\end{array}\right)\left(\begin{array}{c}
i_{1}-1 \\
k-2
\end{array}\right) \prod_{m=2}^{j} 2^{i_{m}}\left(\begin{array}{c}
i_{m}-1 \\
k-1
\end{array}\right),
$$

where the sums are over all integer compositions of $n$ into $j+1$ parts, such that each part, with the exception of the first two parts, has size at least $k$. The first part has size at least $k$ in the first sum, and at least $k-1$ in the second sum.

We also mention that there is a modification of the matching algorithm for the $\mathcal{D}_{n, k}$-arrangement as defined by Björner and Sagan. However, the modification requires making additional rules, and thus is not related to our matching for the general case. We do not give it here, but it will appear in the full version of the paper.

\section{A matching for any $k$-parabolic subspace arrangement}

We now present an algorithm to produce an acyclic matching for the complement of any $k$-parabolic subspace arrangement. First, we fix a finite real reflection group $W$ with corresponding root system $\Phi$. Let $\Delta$ be a simple system in $\Phi$ and $S$ the set of generators corresponding to roots in $\Delta$. Given $I \subseteq \Delta$, we denote by $W_{I}$ the subgroup of $W$ generated by the elements of $I$. Finally, fix a linear order $\left\{s_{1}, \ldots, s_{n}\right\}$ on $S$.

We again consider the Coxeter arrangement $\mathcal{H}(W)$, and the corresponding complex $\Delta_{k}(W)$ from Lemma2.2. We will define an acyclic matching on $\mathcal{F}\left(\Delta_{k}(W)\right)$.

Recall that the length $\ell$ of an element $w$ of $W$ is the length of any reduced expression for $w$ in terms of the generators. Given a coset $v W_{I}$, there is a unique element $u \in v W_{I}$ of minimal length. We let $D(w)=\{s \in S: \ell(w s)<\ell(w)\}$ denote the descents of $w$. Given a set $I$ and a reflection $s \in S$, we let $P(I, s)=\left\{J: J \subseteq I \cup\{s\}, W_{J} \in \mathcal{P}_{n, k}(W)\right\}$. We linearly order $P(I, s)$ by $J \leq K$ if $J \subseteq K$ or $\min (J \backslash K)<\min (K \backslash J)$. Finally, let $I^{\perp}=\{t \in S: s t=t s, \forall s \in I\}$.

Now, consider a coset $u W_{I}$, where $u$ is the element of minimal length in $u W_{I}$. The matching algorithm is as follows:

Let $L=S, X=\emptyset$.

While $L \neq \emptyset$

Let $s=\min L$

If $s \in D(u)$

Set $L=L-s$

Else If $s \in I$

Set $I^{\prime}=I-s$, and Return $u W_{I^{\prime}}$

Else If $P(I, s) \neq \emptyset$

Set $L=(\min (P(I, s))-s)^{\perp}$ And $X=X+s$

Else

End While

Set $I^{\prime}=I+s$, Return $u W_{I^{\prime}}$ 


\section{Return $u W_{I}$}

Given a coset $u W_{I}$, we will refer to the output of the algorithm as $M\left(u W_{I}\right)$. We will let $X\left(u W_{I}\right)$ be the set of elements in $X$ when the algorithm terminates.

If one takes the natural linear order $s_{i}<s_{j}$ for all $i<j$ on $A_{n}$, this algorithm specializes to the one made earlier for the type- $A$ case. If one takes the linear order $s_{j}<s_{i}$ for all $i<j$ on $B_{n}$, this algorithm specializes to the one made earlier for the type- $B$ case.

Proposition 4.1 The algorithm above gives an acyclic matching $M$.

The proof involves using Lemma 2.4 twice. The first time involves the partial order on $W$ that is dual to the right weak order. That is, we take the transitive closure of the cover relations $u \succ u s$ for all $u \in W, s \in S \backslash D(u)$. We will denote the resulting poset by $W^{*}$. The second application of Lemma 2.4 will use the Boolean poset on $S$.

Proof: We only sketch the ideas of the proof. The full version of the paper will have complete details. It is not hard to see that the map $\varphi$ which sends a coset $u W_{I}$ to its minimal coset representative is an order-perserving map between $\mathcal{F}\left(\Delta_{k}(W)\right)$ and $W^{*}$. From properties of minimal coset representatives, one can deduce that for any coset $u W_{I}, \varphi\left(u W_{I}\right)=\varphi\left(M\left(u W_{I}\right)\right)$. Thus, by the Patchwork Lemma, it suffices to show that the algorithm gives an acyclic matching on the fibers of $\varphi$.

So fix an element $w \in W$. Then we attempt to apply 2.4 a second time. For each fiber $\varphi^{-1}(w)$, we consider the map $\psi_{w}$ to the boolean lattice on $S$ given by sending $u W_{I}$ to $X\left(u W_{I}\right)$. A careful study of the algorithm shows that this map is order perserving, and that for any coset $u W_{I} \in \varphi^{-1}(w)$, $\psi\left(u W_{I}\right)=\psi\left(M\left(u W_{I}\right)\right)$. Thus, we only have to show that the algorithm gives an acyclic matching on the fibers of $\psi_{w}$. It is not hard to see that it is a matching. We give an example of the argument for acyclicity.

Figure 2 is an example that the matching is acyclic on the fibers of $\psi_{w}$. We consider $A_{6}$, with $k=3$, $w$ the identity permutation, and the set $X=\left\{s_{1}\right\}$. For type- $A$, the minimum length coset representative for $u W_{I}$ is obtained by refining the set composition so that elements in each block are in increasing order. Every directed cycle has to have alternating edges in the matching. Consider the edge 1/23/4/5/67 $1 / 23 / 45 / 67$. This is an edge in the matching in the fiber $\psi_{w}^{-1}(X)$. Consider trying to make a directed cycle with this edge. Let the next vertex in the directed cycle be $1 / 23 / 45 / 6 / 7$. The next edge in the 'cycle' must be from the matching. However, $1 / 23 / 45 / 6 / 7$ is matched to $1 / 23 / 4 / 5 / 6 / 7$, and as we see in figure 2, this edge is pointed the wrong way. We prove acyclicity by showing that this situation always happens on the fibers of $\psi_{w}$. In general, a matched edge is always of the form $u W_{I} \rightarrow u W_{I+s}$, where $u$ is the coset representative of minimal length, and $s \in S$. Let $t \in I$, and consider $u W_{I+s-t}$, and assume $t$ was chosen so that $X\left(u W_{I+s-t}\right)=X\left(u W_{I}\right)$. Then the algorithm will match $u W_{I+s-t}$ with $u W_{I-t}$, resulting in a picture similar to our figure. Thus, we cannot get a directed cycle in the matching when restricted to $\psi_{w}^{-1}(X)$, for any $w \in W, X \subseteq S$.

We would like to understand the structure of unmatched elements. A simple example will show us that some linear orders on $S$ are more useful than others. For instance, take $A_{5}$ and $k=4$, with linear order $s_{3}<s_{2}<s_{4}<s_{1}<s_{5}, k=4$. Consider the set composition $1 / 23 / 456$, which corresponds to the coset $A_{\left\{s_{2}, s_{4}, s_{5}\right\}}$. Under the algorithm, this coset is unmatched, yet it is of dimension 3 , which is not even. Hence a simple proof of a periodicity condition requires being more specific when picking a linear order for $S$, as the periodicity results followed easily from the linear order we considered previously for $A_{n}$. 


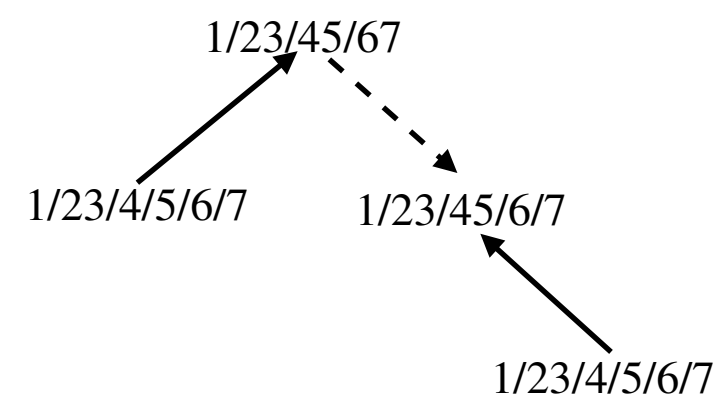

Fig. 2: An example of acyclicity - solid lines are matched edges, dotted lines are not

We restrict ourselves to the case where $W$ is irreducible for a moment. In this case, consider the dynkin diagram $D$ for $W$, and let $P$ be a maximum length path in $D$. Consider a linear order of $S$ such that adjacent vertices of $P$ are adjacent in the order, and the only vertex of $D-P$ is at the end of the linear order, if it exists. A very careful analysis of the algorithm on this linear order reveals that the unmatched cells occur in dimensions that are a multiple of $k-2$.

Now given any finite real reflection group $W$, we fix a linear order on each connected component of its Dynkin diagram in a way similar to above. Then we take a linear extension of these orders, and we see again that unmatched cells occur dimensions that are a multiple of $k-2$.

So combining these observations with Theorem 2.5 and Lemma 2.2, we obtain:

Theorem 4.2 The homology groups $H_{i}\left(M_{\mathcal{W}_{n, k}}\right)$ are non-trivial only when $i=j(k-2)$, for $j \leq\left\lfloor\frac{n}{k}\right\rfloor$. Furthermore, $H_{j(k-2)}\left(M_{\mathcal{W}_{n, k}}\right)$ is free abelian and has rank given by the number of unmatched elements of rank $j(k-2)$ in our matching $M$.

Of course, the case $k=3$ is challenging. Much like in the type- $A$ and type- $B$ case, there are unmatched cells on every level. However, an involution can be used to show that the summation formula in Theorem 2.5. part c, are all zero. Hence the boundary map is the zero map, and the critical cells still index a basis for the homology groups.

We remark that the number of critical cells of dimension 0 is 1 . The only unmatched cell is $w_{0} W_{\emptyset}$, where $w_{0}$ is the element of maximum length in $W$. We also note that for exceptional groups, and arbitrary $k>3$, we can compute the Betti numbers without studying the matching, except possibly the case when $W=E_{8}, k=4$. In all other cases the peridocity conditions, combined with use of group theory to compute the number of cells of $\Delta_{k}(W)$ of dimension $i$, can be used to determine the Betti numbers. A table of these numbers will be included in the full version.

We close with a few open questions. First, similar arguments give a basis for the cohomology. It would be nice to understand the cohomology ring structure in terms of this basis, although this is challenging. Secondly, we note that discrete Morse theory allows us to obtain a minimal cell complex with the same homotopy type as $\mathcal{W}_{n, k}$ for $k>3$. However, with current methods it is complicated to understand the attachment maps of this minimal complex. We do know that, for $k=3$, and large $n$, the resulting complex is not homotopy equivalent to a wedge of spheres, but we currently do not have more information than that. Also, it is still an open problem if the intersection lattice of $\mathcal{W}_{n, k}$ is shellable or not. Even though our approach avoids this question, it would be nice if there was another proof of our results, using 
lexicographic shellability.

Finally, our results have some application to linear decision trees. However, this application no longer has the same simplicity as the $k$-equal problem, and our results do not add anything new to the theory of

linear decision trees. For length considerations, we mention more regarding linear decision trees in the full paper.

\section{References}

[1] Hélène Barcelo and Edwin Ihrig, Lattices of parabolic subgroups in connection with hyperplane arrangements, J. Algebraic Combin. 9 (1999), no. 1, 5-24. MR MR1676736 (2000g:52023)

[2] Hélène Barcelo, Christopher Severs, and Jacob A. White, k-parabolic subspace arrangements, (2009).

[3] Anders Björner and László Lovász, Linear decision trees, subspace arrangements and Möbius functions, J. Amer. Math. Soc. 7 (1994), no. 3, 677-706. MR MR1243770 (95e:52024)

[4] Anders Björner and Bruce E. Sagan, Subspace arrangements of type $B_{n}$ and $D_{n}$, J. Algebraic Combin. 5 (1996), no. 4, 291-314. MR MR1406454 (97g:52028)

[5] Anders Björner and Michelle L. Wachs, Shellable nonpure complexes and posets. I, Trans. Amer. Math. Soc. 348 (1996), no. 4, 1299-1327. MR MR1333388 (96i:06008)

[6] Anders Björner and Volkmar Welker, The homology of " $k$-equal" manifolds and related partition lattices, Adv. Math. 110 (1995), no. 2, 277-313. MR MR1317619 (95m:52029)

[7] Eva Maria Feichtner and Dmitry N. Kozlov, On subspace arrangements of type D, Discrete Math. 210 (2000), no. 1-3, 27-54, Formal power series and algebraic combinatorics (Minneapolis, MN, 1996). MR MR1731606 (2001k:52039)

[8] Mark Goresky and Robert MacPherson, Stratified Morse theory, Ergebnisse der Mathematik und ihrer Grenzgebiete (3) [Results in Mathematics and Related Areas (3)], vol. 14, Springer-Verlag, Berlin, 1988. MR MR932724 (90d:57039)

[9] Dmitry Kozlov, Combinatorial algebraic topology, Algorithms and Computation in Mathematics, vol. 21, Springer, Berlin, 2008. MR MR2361455 (2008j:55001)

[10] Dmitry N. Kozlov, General lexicographic shellability and orbit arrangements, Ann. Comb. 1 (1997), no. 1, 67-90.

[11] Peter Orlik, Complements of subspace arrangements, J. Algebraic Geom. 1 (1992), no. 1, 147-156. 